\title{
Updates and Challenges in Refractive Lens Exchange
}

An Expert Interview with Deepinder K Dhaliwal

University of Pittsburgh School of Medicine, UPMC Eye Center and Charles T Campbell Ocular Microbiology Laboratory, Pittsburgh, PA, US

DOl: https://doi.org/10.17925/USOR.2018.11.1.17

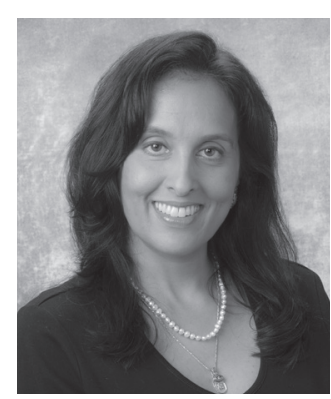

\section{Deepinder K Dhaliwal}

Deepinder K Dhaliwal MD, L.AC, is a Professor at the University of Pittsburgh School of Medicine, and Director of Refractive Surgery and the Director of the Cornea Service at the UPMC Eye Center. Dr Dhaliwal is also the Founder and Director of the Center for Integrative Eye Care at the University of Pittsburgh. She became a licensed acupuncturist in 2006 and is systematically researching alternative treatments for eye disease.

Dr Dhaliwal is a recognized expert in her field and teaches corneal and refractive surgical techniques to other ophthalmologists globally. In addition to teaching and research activities, she has authored several book chapters, numerous journal articles, and serves on the editorial board of several ophthalmology journals. In recognition of her clinical and surgical skills she has been selected as a 'Top Doctor' by her peers every year since 2006.

\section{Keywords}

Refractive lens exchange, refractive surgery, laser-assisted in situ keratomileusis (LASIK), photorefractive keratectomy (PRK), IOL

Disclosure: Deepinder K Dhaliwal has nothing to disclose in relation to this article.

Review Process: This is an expert interview with an Editorial Board member and as such did not undergo the journal's standard peer review procedure.

Authorship: All named authors meet the International Committee of Medical Journal Editors (ICMJE) criteria for authorship of this manuscript, take responsibility for the integrity of the work as a whole, and have given final approval to the version to be published.

open Access: This article is published under the Creative commons Attribution Noncommercial License, which permits any noncommercial use, distribution, adaptation, and reproduction provided the original author(s) and source are given appropriate credit. (c) The Authors 2018.

Received: March 17, 2018

Published Online: March 26, 2018

Citation: US Ophthalmic Review, 2018;11(1):17-18

Corresponding Author: Deepinder Dhaliwal, 203 Lothrop Street, Room 759, Pittsburgh 15213, PA, US. E:dhaliwaldk@upmc.edu

Support: No external funding was received in the publication of this article.
$\mathrm{R}$ efractive lens exchange (RLE) is frequently used as a refractive surgical procedure for the correction of high presbyopia and high hyperopia, for which laser-assisted in situ keratomileusis (LASIK), photorefractive keratectomy (PRK) or phakic intraocular lens (IOL) refractive surgery is unsuitable. In an expert interview, Deepinder K Dhaliwal, Professor of Ophthalmology at the University of Pittsburgh School of Medicine, Director of Cornea and Refractive Surgery at the UPMC Eye Center, and Associate Medical Director of the Charles T Campbell Ocular Microbiology Laboratory, discusses the challenges of refractive lens exchange and other aspects of refractive surgery.

\section{Q. Which patients are unsuitable for refractive lens exchange?}

It is tempting for anterior segment surgeons to consider refractive lens exchange in any presbyopic patient. However, highly myopic patients with a clear lens who undergo refractive lens exchange have an unacceptably high rate of retinal detachment. If these patients are not candidates for LASIK or PRK because of a corneal thickness limitation, a phakic IOL seems to be a better option as long as there is an adequate anterior chamber depth. Conversely, hyperopic patients generally tolerate refractive lens exchange well and the procedure can be especially helpful if there is a crowded anterior segment.

\section{Q. What are the most common complications of refractive lens exchange?}

In the young myope, retinal detachment is a risk, as discussed above. A study in France demonstrated that the risk of retinal detachment was $9.2 \%$ at 4 years in high myopes. ${ }^{1}$ It is also important to consider the risk of intraocular infection, for which prophylaxis is essential. The correct application of povidoneiodine preoperatively is critically important and we are not paying enough attention to that simple step. The literature supports that the correct method of application is to squirt $5 \%$ povidone iodine into the upper and lower fornices in order to bathe the lids and the entire ocular surface with the antiseptic, and then leave it untouched and undiluted for at least 3 minutes. We then apply sterile lidocaine gel and the patient is then taken into the operating room. The nurses in the operating room then perform a second prep of the ocular surface and we carefully drape so that lids and lashes are completely covered and isolated from the surgical field. Postoperatively, we use a collagen shield soaked in antibiotic along with topical antibiotic drops rather than an intracameral or intravitreal antibiotic injection. Using this protocol, we have had a zero incidence of endophthalmitis over the past 20 years. Other risks include not meeting the refractive endpoint and unwanted optical aberrations (such as glare and halo) that could be inherent in some IOL designs. Preoperative discussion should be detailed. As long as the patient's postoperative result meets or exceeds their preoperative expectations, the patient should be satisfied. 


\section{Q. What are the major challenges posed by irregular corneas when correcting astigmatism with a toric IOL?}

Toric IOLs are designed to correct regular astigmatism. However, if the patient has a component of irregular astigmatism as well then there are a few key issues to consider. If the patient was satisfied with the quality of vision while wearing spectacles (which only correct the regular component of the astigmatism), then it is likely that a toric IOL will provide an adequate result. However, if a patient is dependent on gas-permeable contact lenses to obtain sharp vision then correction of only the regular astigmatism with a toric IOL will not replicate the optics and the patient will likely be unhappy with the result since the irregular component of the astigmatism will be uncorrected. They need to understand that they will be unable to wear gas-permeable contact lenses after the toric IOL implantation (since that would unmask the astigmatic power of the lens implant). It is Important to thoroughly assess the etiology of astigmatism before cataract surgery and consider preoperative correction of treatable causes of irregular astigmatism, for example from epithelial basement membrane dystrophy. In such cases we perform superficial keratectomy, let the cornea heal, and then re-check the keratometry after 6-8 weeks. A toric lens can then fully correct any residual regular astigmatism. If, however, patients have irregular astigmatism from an ectatic disorder such as keratoconus, and they are dependent on rigid contact lenses for optimal vision, I would not implant a toric lens if they are going to wear contact lenses postoperatively. If they are elderly and unwilling to wear contact lenses, I will offer correction of the regular astigmatism with a toric lens as long they understand that their irregular astigmatism will remain.

\section{Q. When would you consider using a piggyback IOL?}

I would reserve use of a piggyback IOL for patients who have a large refractive surprise postoperatively, and are not candidates for correction with spectacles, contact lenses, or laser vision correction. I do not use them in primary cases. When performing surgery on patients with high hyperopia, I prefer to implant the high power IOL in the capsular bag rather than splitting the power with a piggyback IOL. We have limited options of piggyback lenses in the US. $\square$

1. Daien V, Le Pape A, Heve D, et al. Incidence, Risk Factors, and Impact of Age on Retinal Detachment after Cataract Surgery in France: A National Population Study. Ophthalmology. 2015;122:2179-85. 